University of Nebraska - Lincoln

DigitalCommons@University of Nebraska - Lincoln

P. F. (Paul Frazer) Williams Publications

Electrical \& Computer Engineering, Department

April 1985

\title{
A new design concept for field distortion trigger spark gaps
}

G. Schaefer

Texas Tech University, Lubbock, Texas

B. Pashale

Texas Tech University, Lubbock, Texas

P. F. Williams

University of Nebraska - Lincoln, pfw@moi.unl.edu

K. H. Schoenbach

Texas Tech University, Lubbock, Texas

H. Krompholz

University of Nebraska - Lincoln

Follow this and additional works at: https://digitalcommons.unl.edu/elecengwilliams

Part of the Electrical and Computer Engineering Commons

Schaefer, G.; Pashale, B.; Williams, P. F.; Schoenbach, K. H.; and Krompholz, H., "A new design concept for field distortion trigger spark gaps" (1985). P. F. (Paul Frazer) Williams Publications. 19.

https://digitalcommons.unl.edu/elecengwilliams/19

This Article is brought to you for free and open access by the Electrical \& Computer Engineering, Department of at DigitalCommons@University of Nebraska - Lincoln. It has been accepted for inclusion in P. F. (Paul Frazer) Williams Publications by an authorized administrator of DigitalCommons@University of Nebraska - Lincoln. 


\title{
Anew design concept for field distortion trigger spark gaps
}

\author{
G. Schaefer, B. Pashaie, P. F. Williams ${ }^{\text {a) }}$, K. H. Schoenbach, and H. Krompholz \\ Department of Electrical Engineering/Computer Science, Texas Tech University, Lubbock, Texas 79409
}

(Received 16 August 1984; accepted for publication 30 October 1984)

\begin{abstract}
A common field distortion triggered spark gap utilizing geometric field enhancement at sharp edges usually operates in a cascade mode via the trigger electrode. A new trigger concept is proposed allowing strong field enhancement and direct breakdown between the two main electrodes. A test setup was designed to prove the feasibility of this concept. Experimental results on delay and jitter depending on percent breakdown voltage are presented. Best results achieved are a delay of $9 \mathrm{~ns}$ and a jitter of $2 \mathrm{~ns}$ at a self-breakdown voltage of $15 \mathrm{kV}$.
\end{abstract}

\section{INTRODUCTION AAD TRIGGER CONCEPT}

Spark gaps using field distortion triggering are initially designed to provide hold-off voltage without trigger, and a trigger electrode shaped and located on an equipotential surface in the gap is then added. Triggering is accomplished by abruptly changing the potential of this electrode, thereby increasing the field between the trigger electrode and one of the gap electrodes. A typical example is the three electrode gap with a blade as a midplane trigger electrode located approximately half way between the main electrodes. ${ }^{1}$ In the hold-off state the blace is in the plane of an equipotential and no field enhancement is generated at the edge of the blade. By changing the potential of the trigger electrode a very strong field enhancement at the edge can be produced. Since the maximum field enhancement occurs at the trigger electrode, however, the switch operates usually in a cascade mode in which the gap between one electrode and the trigger electrode is first closed (initiated by the trigger pulse) and then the second half of the gap is closed by the voltage across the switch.

To allow for geometrically enhanced field distortion and still to avoid cascade breakdown, field enhancement at an edge of one of the main electrodes can be used. This edge, however, must also be shielded in the hold-off state of the gap. ${ }^{2}$ A schematic diagram of a spark gap based on this concept is shown in Fig. 1. In this device the trigger electrode is used to shape the electric field intensity in the gap in both the hold-off state and the triggering state. In the hold-off state the trigger electrode is kept at the same potential as electrode (1) and its surface towards the gap is shaped to minimize the geometric field enhancement effects at the main gap electrode, thereby maximizing the hold-off voltage. In the triggering state the potential of the trigger electrode is moved towards the potential of electrode (2). The trigger electrode subsequently serves to enhance the field, providing improved triggering, in two ways: moving the equipotential toward one gap electrode, and simultaneously turning on the geometric field enhancement. Such a trigger concept combines several advantages: lized.

(1) Geometrically enhanced field distortion can be uti-

(2) The strongest field enhancement occurs at one of the

\footnotetext{
A) P. F. Williams is now with the University of Nebraska, Department of
} Electrical Engineering, Lincoln, Nebraska 68508. main electrodes, and breakdown, without cascading via the trigger electrode, is possible.

(3) Since the electrode can be shape without changing the hold-off performance, the field enhancement at a main electrode can be much larger than in common field distortion triggering.

(4) Shape and surface conditions of this main electrode do not determine the hold-off performance of the gap, making the gap more independent of erosion.

This concept would have to be applied to both main electrodes for protection of both.

\section{TEST SETUP}

The experimental setup used to test this trigger concept is shown in Fig. 2. A parallel plane line was used as charging and transmission line (total impedance $\sim 12.5 \Omega$ ). The switch consisted of eight individual gaps. The upper conductor of the lines was divided into eight individual stripes to provide for transit time insulation of the individual gaps (im-

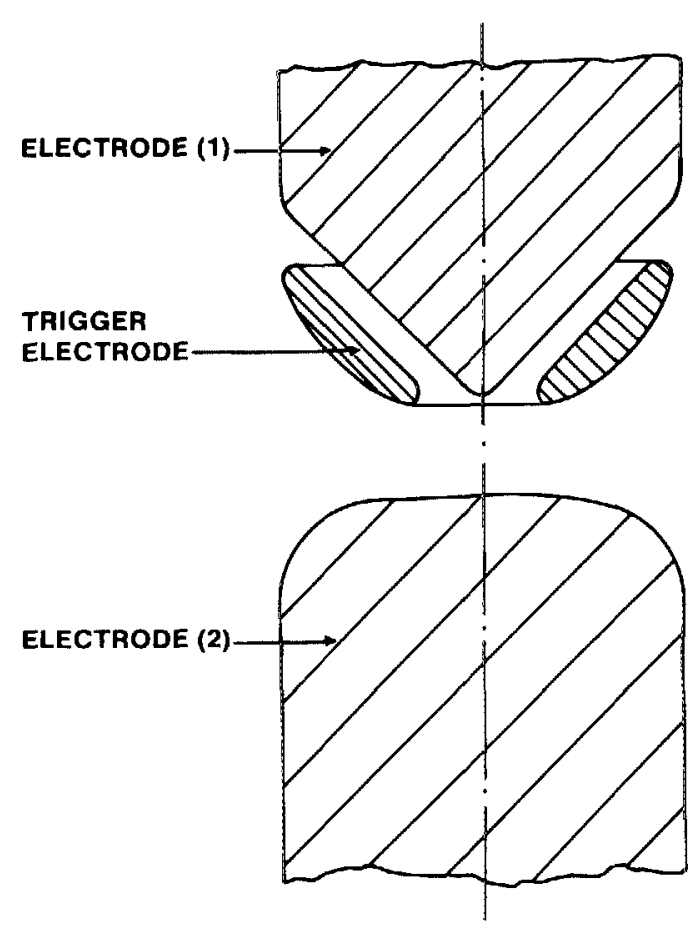

FIG. 1. Schematic diagram of a spark gap with geometrically enhanced field distortion at the main electrode. 


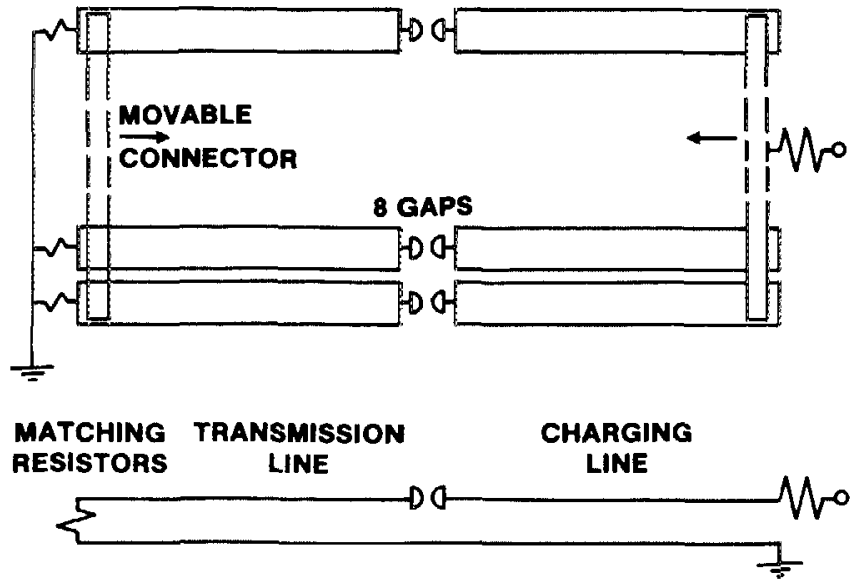

FIG. 2. Experimental setup.

pedance/stripe $\sim 100 \Omega$ ). The time constant for the transit time insulation could be varied in the range of $0-5 \mathrm{~ns}$ by moving metal bars connecting the individual transmission and charging lines.

Two different electrode configurations were used as shown in Fig. 3. The configuration (A) uses one triangular shaped main electrode (1) with a pair of two rods as trigger electrodes and one rounded main electrode (2), while the configuration (B) uses a symmetric configuration with two triangular main electrodes. The trigger electrodes in any case were pairs of rods triggering all eight individual gaps at the same time as demonstrated for the configuration $(B)$ in Fig. 4. Bare stainless steel bars as well as bars covered with a dielectric material (glass tubes or epoxy) or with a resistive material (graphite-filled epoxy) have been used.

The trigger circuits are shown in Fig. 5. The trigger pulse was provided by a secondary gap which was operated in the self-breakdown mode and the breakdown voltage was adjusted through changing the secondary gap electrode separation. In the hold-off state the trigger electrodes are at the potential of the adjacent main electrode. When the secondary gap fires the potential of the trigger electrode is driven towards the potential of the opposite electrode.

For the circuits (A), (B), and (C) the full charging voltage of the line can be applied to the trigger electrode, while for the circuit $(D)$ both trigger electrodes potentials move towards the midplane potential of the gap.
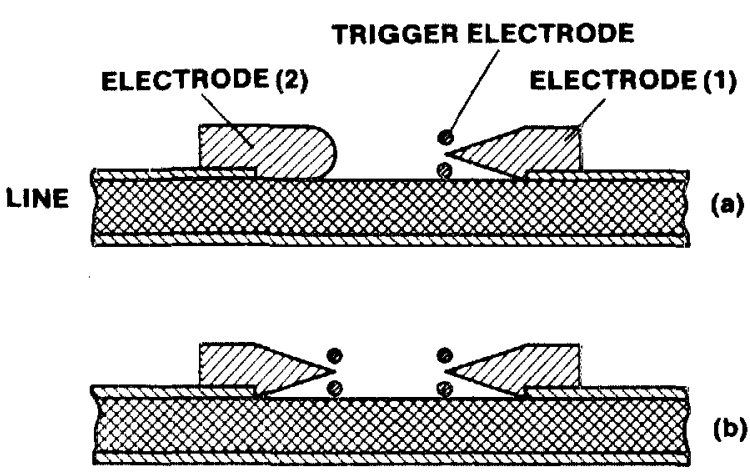

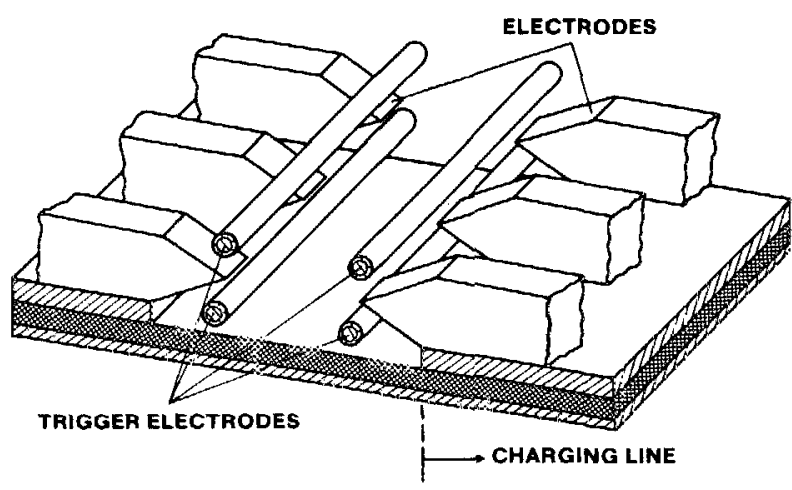

FIG. 4. Trigger electrode arrangement.

\section{EXPERINEENTAL RESULTS}

The experiments performed concentrated on the spark gap performance with respect to delay and jitter depending on the applied voltage in percent of the breakdown voltage. The first experiments to determine the optimum type of trigger electrode and polarity were performed with an electrode geometry as shown in Fig. 3(a) and a circuit as shown in Fig. 5 (a). Although the system could be triggered with either polarity, clearly better trigger results were obtained with the electrode (1) being at positive potential and the trigger electrode being driven towards a negative potential. Triggering

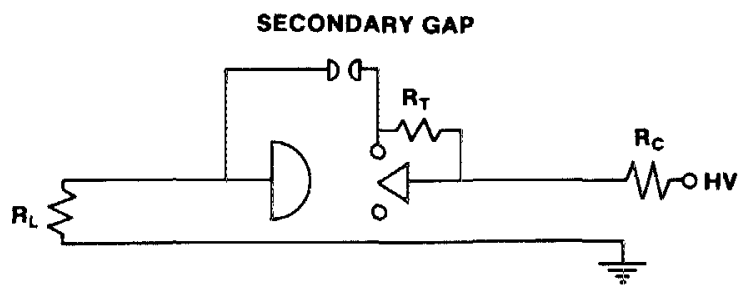

(a)

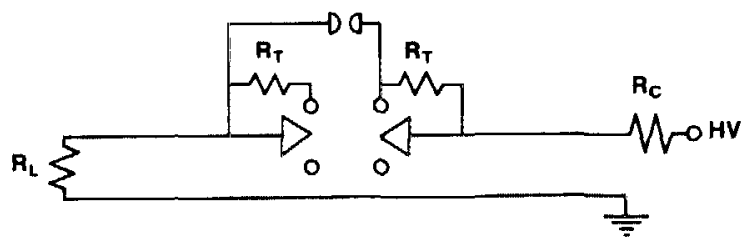

(b)

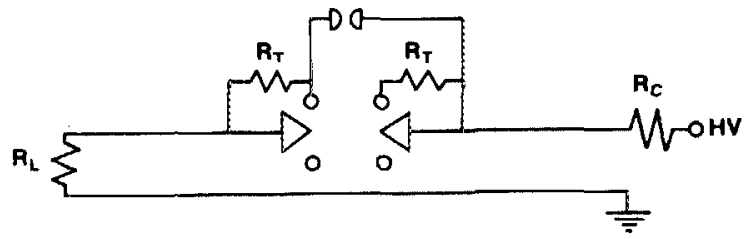

(c)

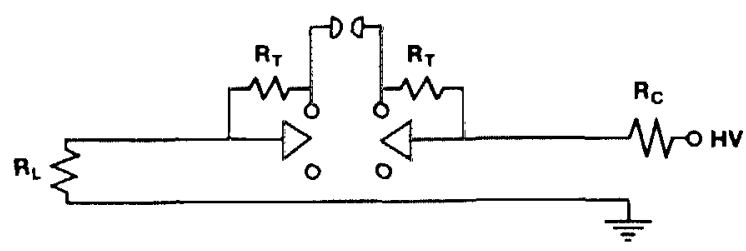

(d)

FIG. 3. Electrode geometrics.

FIG. 5. Trigger circuits. 
was possible with all types of trigger electrodes used. Bare metal rods as trigger electrodes had the disadvantage that a very precise symmetric alignment was necessary to avoid arcs between the trigger electrode and the main electrode. The best results were obtained with electrodes covered with a dielectric layer (glass or epoxy). Since the system performance did not depend on repetition rate $\left(10^{-2}-1 \mathrm{~Hz}\right)$ surface charges on the surface of the dielectric seemed not to affect the performance of the trigger method at these low repetition rates. Surface charges could be eliminated with resistive layers instead of a dielectric, but arcing to the trigger electrode again required precise alignments unless layers with high resistivity were used (thickness $\sim 0.5 \mathrm{~mm}$, resistivity $\sim 10^{6}$ $\Omega \mathrm{cm}$ ).

The circuits in Figure $5(\mathrm{~b})$ and $5(\mathrm{c})$ are equivalent to circuit (A) since only one pair of trigger electrodes changes its potential. No significant differences in the performance of the spark gaps was realized for these circuits as long as the right polarity was used. The performance of the gap with the circuit shown in Figure 5(d) was significantly worse with respect to delay and jitter.

The optimum position of the trigger electrodes was de-

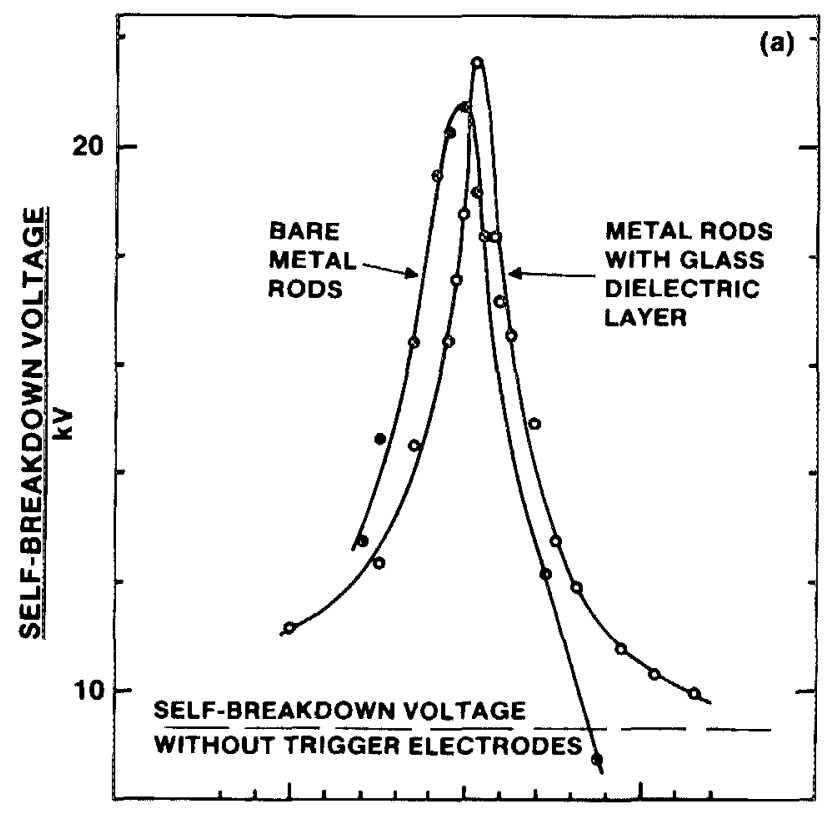

TRIGGER ELECTRODE POSITION (1 mm/div)

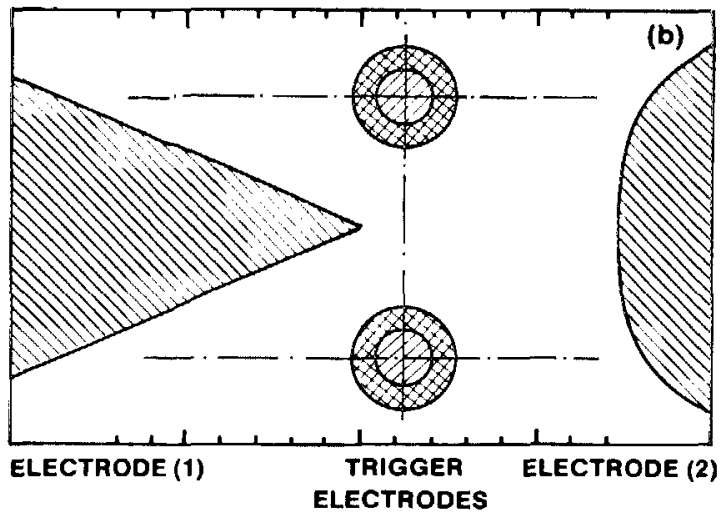

FIG. 6. Self-breakdown voltage as a function of trigger electrode position (a) and electrode geometry (b). termined through measurements of the self-breakdown voltage shown in Fig. 6(a). In these experiments the pair of trigger electrodes was moved in the direction of the interelectrode spacing as shown in Fig. 6(b). The results clearly show the shielding of the edged main electrode resulting in an increase of the self-breakdown voltage of more than a factor of 2 compared to the gap without trigger electrodes. For optimum shielding no significant difference was observed for the two different types of trigger electrodes. The maximum self-breakdown voltage observed is nearly the uniform field breakdown value.

The following measurements on the trigger performance were obtained with the circuit in Fig. 5(a) and 5(d) and a positive charging voltage. All experiments are performed in atmospheric air. The rise time of the trigger pulse was of the order of $12 \mathrm{~ns}$. Delay and jitter were determined by measuring the time between the voltage rise at the trigger electrode and at the main electrode. Figure 7 shows the delay depending on percent self-breakclown voltage $\left(\% V_{\mathrm{sB}}\right)$ for the two circuits. It should be pointed out that the maximum voltage of the trigger pulse always equals the charging voltage in the circuit used. So with a decreasing value of $\% V_{\mathrm{SB}}$ the maximum voltage of the trigger pulse automatically decreased.

As demonstrated in Fig. 7, a minimum delay time of 9 ns was achieved with circuit Fig. 5(a) for a self-breakdown voltage of $15 \mathrm{kV}$. Above $90 \% V_{\mathrm{SB}}$ self-breakdown the delay does not significantly change with $\% V_{\mathrm{SB}}$ as required for

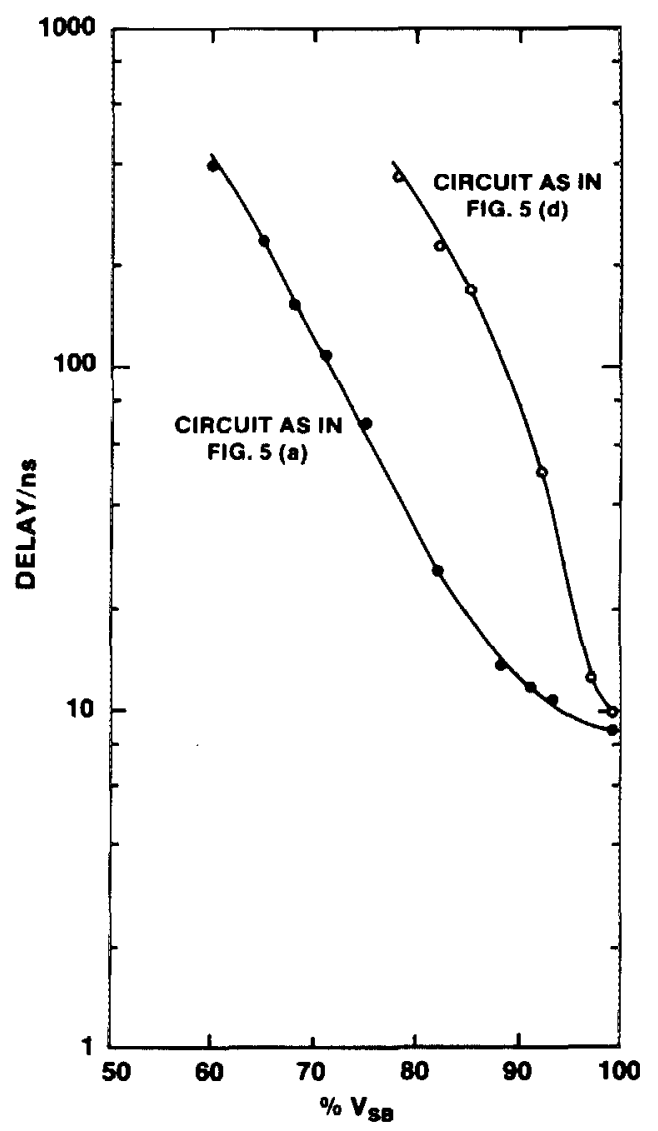

FIG. 7. Delay vs \% self-breakdown voltage for two trigger circuits $\left(V_{\mathrm{SB}}=15 \mathrm{kV}\right)$. 


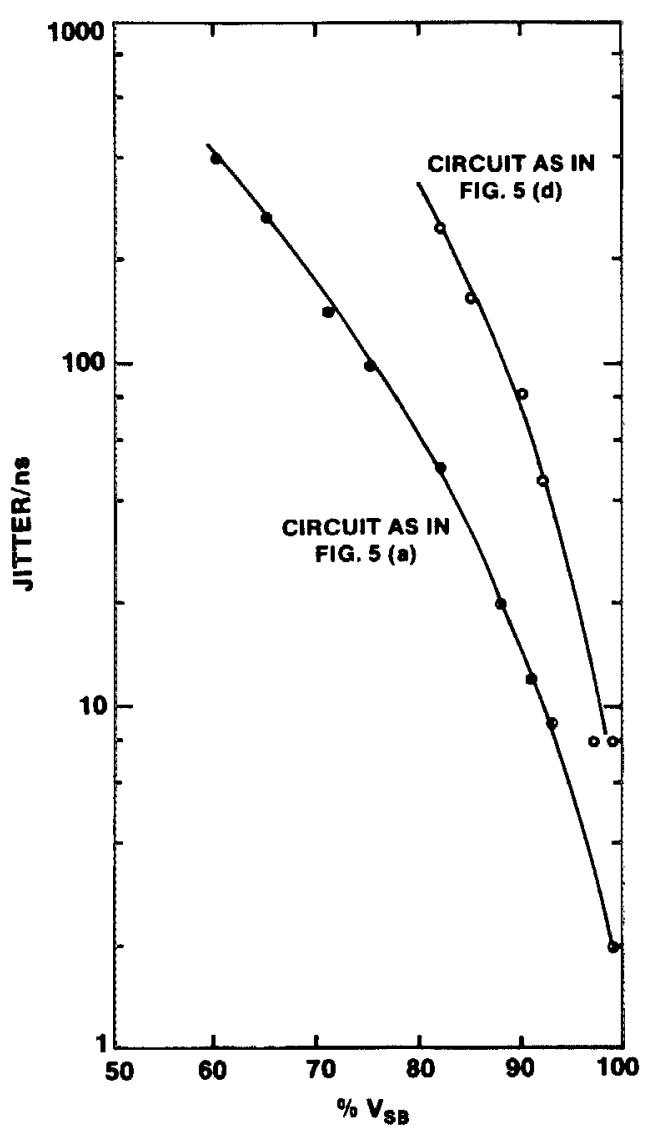

FIG. 8. Jitter vs $\%$ self-breakdown voltage for two trigger circuits $\left(V_{\mathrm{SB}}=15 \mathrm{kV}\right)$.

multichanneling or parallel triggering of several gaps.

Figure 8 shows the jitter depending on $\% V_{\mathrm{SB}}$ for the same operation conditions as in Fig. 7. The jitter shown here is the maximum jitter in a series of 20 shots. Close to $100 \%$ $V_{\mathrm{SB}}$ a jitter of $\sim 2 \mathrm{~ns}$ could be achieved.

These results were also proven through parallel operation of the eight gaps with one pair of trigger electrodes for all gaps as shown in Fig. 4. With a transit time insulation of 5 ns, parallel triggering of all gaps was achieved if the charging voltage was kept above $95 \% V_{\text {SB }}$. Fine adjustment of the self-breakdown voltage of each gap was difficult, however, and it is likely that some gaps were operated at significantly lower values of $\% V_{\text {SB }}$.

\section{DISCUSSION}

The exploratory experiments demonstrate the feasibility of the proposed field distortion trigger concept. Results on delay time and jitter indicate that this method may be suitable for multichanneling and the parallel operation of spark gaps. Field code calculations are required to optimize the geometry for a maximum hold-off voltage in the off-state and maximum field enhancement in the on-state. Further experiments are required with a test gap allowing operation in different gases with variable pressure and a trigger circuit allowing the independent variation of $\% V_{\mathrm{SB}}$ and trigger pulse parameters.

The physical mechanisms responsible for triggering are of interest. Referring to Fig. 1, in the triggered state with the trigger electrode connected electrically to electrode (2), a very high electric field exists in the vicinity of electrode (1), while a much reduced field is produced in the main gap region between the trigger electrode and electrode (2). Two mechanisms for triggered breakdown seem possible. In the first, a streamer is launched inside the high field region, and propagates past the trigger electrode into the low applied field region. Propagation continues because the streamer body forms a weakly conducting needle connected to electrode (1), thereby producing high electric fields in the vicinity of its tip. After the streamer has traversed the gap, ohmic heating occurs and converts the weakly conducting channel left by the streamer into an arc channel.

In the second case, initial breakdown occurs through a purely Townsend mechanism. In this case, the criterion for breakdown is that sufficient electron avalanche multiplication occurs so that one electron leaving the cathode may reproduce itself at the cathode through the avalanching and other appropriate secondary processes. Here, the relevant quantity is the electron amplification due to impact ionization, $A=\int_{0}^{d} \alpha(E) d x$, where $E=E(x)$ is the applied field, assuming no space-charge distortion. $E$ is subject to the constraint $\int_{0}^{d} E(x) d x=V$, where $V$ is the gap voltage. Since the impact ionization coefficient $\alpha$ is a strongly increasing function of field for fields around the breakdown field, the amplification factor $A$ will be much larger for the highly nonuniform field produced in the triggering state than for the uniform field produced when the triggering electrode is connected to electrode (1). Thus, according to the Townsend criterion, the gap may be strongly overwolted in the triggered configuration, while remaining undervolted in the normal configuration.

The experimental data on delay suggest that both mechanisms occur. For applied voltages near the static breakdown voltage, the delay is found to be approximately $10 \mathrm{~ns}$. Considering the substantially reduced field in the region between the triggering electrode and electrode (2), electrons emitted from the cathode [electrode (2)] would require $\sim 50$ ns to traverse the gap. Thus, it seems difficult to explain delay times less than about 100 ns with the Townsend mechanism. At the opposite extreme, delays approaching $1 \mu \mathrm{s}$ are observed for low applied voltages. Even considering the dielectric relaxation time required for a streamer to produce the high field enhancements needed in this regime, a streamer transit time exceeding 100 ns seems unlikely. Additional time is required, of course, to convert this streamer channel into an arc channel but this time should not be a strong function of the applied voltage, and considering the $10 \mathrm{~ns}$ delay observed at $95 \% V_{\mathrm{SB}}$, should not exceed several tens of nanoseconds at $50 \% V_{\mathrm{SB}}$. Thus the low voltage data suggest that a Townsend mechanism is at work. The two mechanisms, streamer and Townsend, are not incompatible, and it is likely that there is continuous transition from one to the other as the gap voltage is reduced.

\section{PERSPECTIVES}

The proposed trigger concept is well suited to combine field distortion with other trigger concepts to improve the switch performance. The important feature of this concept 
here again is that the field enhancement occurs close to the surface of one main electrode and that this main electrode is partially shielded from the field in the hold-off state.

For trigatrons, it is well known that delay and jitter are drastically improved by overvolting the gap. Subsequently the combination of a trigatron trigger in the main electrode and a field enhancement generated by a field distortion in volume close to this trigatron electrode could provide for the same condition without the need to overvolt the total gap. The same considerations also hold for those laser triggered gaps where the laser spark is produced at or close to the surface of one main electrodes. The combination of the pro- posed field distortion concept with one of these trigger methods would therefore provide significantly improved performance in an undervolted main gap.

\section{ACKNOWLEDGMENTS}

This work was supported jointly by the Air Force Office of Scientific Research and the Army Research Office.

${ }^{1}$ A. E. Bishop and G. D. Edmonds, Proc. IEE 113, 1549 (1966).

${ }^{2}$ J. K. Hepworth, R. C. Klewe, and B. A. Tozer, Proc. IEE 119, 1751 (1972). 